\title{
LOS ROLES, TIPO DE DECISIONES Y HABILIDADES GERENCIALES DE LOS GERENTES DE OCAÑA
}

\section{THE ROLES, TYPE OF DECISIONS AND MANAGERIAL SKILLS OF THE MANAGERS OF OCAÑA}

\author{
MSc. Sugeidy Contreras Suarez ${ }^{\text {a }}$, Est. Karen Tatiana Sarabia Vergel ${ }^{\text {b, }}$ Est. Yeraldin Sarabia \\ Vergel $^{\mathrm{c}}$ \\ ${ }^{a}$ Universidad Francisco de Paula Santander Ocaña, CIDFE, Vía Acolsure, Sede el Algodonal a, \\ Ocaña, Colombia, scontrerass@ufpso.edu.co \\ ${ }^{\mathrm{b}}$ Universidad Francisco de Paula Santander Ocaña, Vía Acolsure, Sede el Algodonal a, Ocaña, \\ Colombia, ktsarabiav@ufpso.edu.co \\ ${ }^{\mathrm{c}}$ Universidad Francisco de Paula Santander Ocaña, Vía Acolsure, Sede el Algodonal, Ocaña, \\ Colombia, ysarabiav@ufpso.edu.co
}

Fecha de recepción: 01-04-2017

Fecha de aprobación: 15-06-2017

\begin{abstract}
Resumen: El presente artículo es producto de una investigación, cuyo objetivo consistió en el estudio de los roles, tipo de decisiones y habilidades gerenciales que tienen los gerentes de las empresas de la ciudad de Ocaña, en el que permitió conocer el perfil, conocimientos, habilidades, experiencia y actitudes, al igual que el estilo gerencial en las organizaciones, como puede hacerla rentable y enfrentar los retos de un mundo globalizado, en una administración compleja, con incertidumbres y desafíos propios del Siglo XXI. Con esta investigación se establecieron características que poseen los gerentes, permitiendo conocer los diferentes aspectos que tienen estos para dirigir sus empresas y de igual forma saber cómo influye el perfil y el estilo gerencial en el direccionamiento de los recursos, ya que la nueva economía necesita organizaciones eficientes y competitivas, enfrentando los desafíos del actual entorno. Se debe decir que, para lograr la identificación de estos aspectos, se aplicó en la metodología el tipo de investigación descriptiva, con una población objeto de 5380 empresas, en donde se llevó a cabo el diseño y aplicación de la encuesta a los representantes legales de las empresas de la cuidad de Ocaña, logrando establecer los diferentes objetivos planteados y así mismo desarrollarlos.
\end{abstract}

Palabras clave: actitudes, conocimientos, estrategias, habilidades gerenciales, tipo de decisiones, roles. 


\begin{abstract}
The present article is the product of an investigation, whose objective was to study the roles, type of decisions and managerial abilities that have the managers of the companies of the city of Ocaña, in which it allowed to know the profile, knowledge, abilities, Experience and attitudes, as well as the management style in organizations, how can it make it profitable and face the challenges of a globalized world, in a complex administration, with uncertainties and challenges typical of the 21 st Century. This research established the characteristics that managers possess, allowing them to know the different aspects that these have to manage their companies and also to know how the profile and management style influences the resource management, since the new economy needs organizations Efficient and competitive, facing the challenges of the current environment. It must be said that, in order to achieve the identification of these aspects, the methodology was applied to the type of descriptive research, with a target population of 5380 companies, where the design and application of the survey was carried out to the legal representatives of The companies of the city of Ocaña, managing to establish the different objectives and develop them.
\end{abstract}

Keywords: Attitudes, knowledge, strategies, managerial skills, type of decisions, roles

\section{INTRODUCCIÓN}

Hoy día, las organizaciones se encuentran en un contexto heterogéneo empresarial, caracterizado por fenómenos como la globalización, la competitividad, las nuevas tecnologías y la proliferación de entornos complejos e impredecibles. Un contexto de cambio continuo, que obliga a las organizaciones a adaptarse para competir, tanto en el mercado internacional, como en los mercados internos. (Larocca, 2010), Ante esta situación, las organizaciones deben actuar más allá de los sistemas tradicionales de gestión, los cuales siguen siendo válidos pero insuficientes, y deben buscar factores de diferenciación sostenible en el tiempo y difíciles de imitar por parte de sus competidores. (Ferrer \& Clemenza, 2006)

Por consiguiente, se realizó este trabajo con el fin de identificar los roles, tipo de decisiones y habilidades gerenciales que tienen los gerentes de las empresas de la ciudad de Ocaña, se desarrollaron objetivos como conocer los roles, habilidades y destrezas, que caracterizan el gerente, su formación, conocimientos y experiencias gerenciales requeridas, como también analizar cómo influye el perfil y el estilo gerencial en el direccionamiento de los recursos y por ultimo establecer qué tipo de decisiones y estrategias toman los gerentes. (Duarte, 2013)

Para la investigación se tuvo en cuenta el marco referencial compuesto por el marco histórico, conceptual, teórico y legal, al igual que un diseño metodológico y una población de 254 empresas de la ciudad de Ocaña, Norte de Santander, a las que se les aplico una encuesta que permitió el desarrollo de los objetivos y así realizar unas conclusiones y recomendaciones de la investigación.

\section{METODOLOGÍA}

\section{Tipo de investigación}

Para la realización de esta investigación se utilizó el tipo de investigación no experimental de alcance descriptivo, ya 
que se logra conocer las variables dependientes del estudio, es decir, rol, estilo, habilidades, competencias, liderazgo, toma de decisiones por parte de los gerentes, por otra parte, lograr con la misma investigación una caracterización básica sobre la formación, conocimiento y experiencia del gerente que labora actualmente en las empresas de Ocaña. (Guzman, 2014)

\section{Población}

En la investigación, la población objeto de estudio estuvo conformada por 5380 empresas, información obtenida directamente desde la Cámara de Comercio de la ciudad de Ocaña, Norte de Santander.

\section{Muestra}

Por considerarse la población demasiado numerosa, se estimó conveniente aplicar la siguiente formula (Herrera, 2011), para poder establecer el tamaño de la muestra:

$$
n=\frac{(Z)^{2} * N * p * q}{(E)^{2}(N-1)+(Z)^{2} * p * q}
$$

Dónde:

$\mathrm{n}=$ Muestra

$\mathrm{N}=$ Población dada en el estudio $=5380$

$\mathrm{Z}=$ Indicador de Confianza $=95 \%=1.96$

$\mathrm{p}=$ Proporción de aceptación $=50 \%=0.5$

$\mathrm{q}=$ Proporción de rechazo $=50 \%=0.5$

$\mathrm{E}=$ Error poblacional dispuesto a asumir

$6 \%=0.06$

$$
n=\frac{(1.96)^{2} *(5380) *(0.5) *(0.5)}{(0.06)^{2}(5380-1)+(1.96)^{2} *(0.5) *(0.5)}
$$

$n=\frac{5167}{20.32}$ $n=254$

Técnicas e instrumentos de recolección de la información.

La investigación llevo a cabo el diseño y aplicación de la encuesta, técnica e instrumento dirigida a los representantes legales, directivos o administradores de las empresas de la ciudad de Ocaña, Norte de Santander.

Técnicas de recolección y análisis de la información.

Luego de aplicada la encuesta, la información se analizó cuantitativamente a través de tablas y gráficas, después de eso, se hará el análisis cualitativo de cada una de las respuestas, siendo datos primarios. En cuanto a los datos secundarios partirán de las búsquedas por internet, bibliotecas, archivos, documentos que tengan relación con la investigación, por otra parte, luego de aplicada la encuesta.

\section{RESULTADOS}

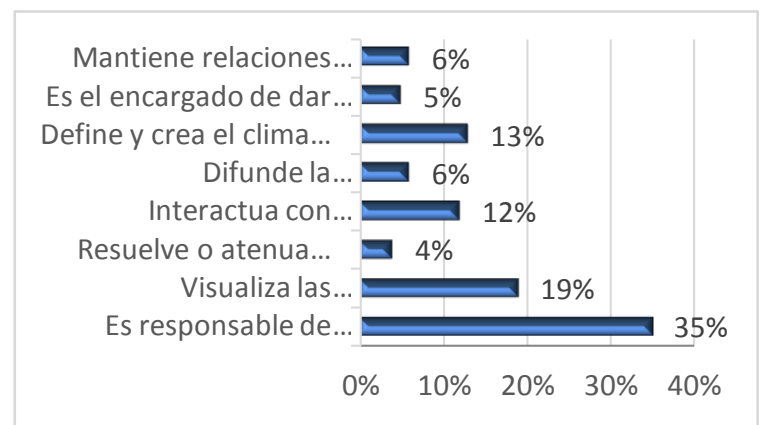

Figura 1. Roles gerenciales empresas de la ciudad de Ocaña.

Fuente: Información suministrada por las empresas de la ciudad de Ocaña. 
Siendo los roles gerenciales una categoría de comportamientos que se van adquiriendo, se puede decir, que el $35 \%$ son responsables de recolectar, mantener y procesar toda la información que es de utilidad para la organización, teniendo en cuenta que este pertenece a la clasificación de roles informacionales, el cual corresponde al rol monitor, por otra parte el $19 \%$ se encargan de visualizar oportunidades e iniciar procesos de innovación y cambio, permitiéndoles ser más competitivos $\mathrm{y}$ tener mejores resultados, siendo factores importantes para mantener la empresa en el mercado; de igual forma el $13 \%$ define y crea el clima de buenas relaciones entre los miembros de la organización, es decir ser un buen líder, lo que hace que haya un buen trabajo en equipo y un $12 \%$ interactúa con personas y organizaciones externas, siendo de gran apoyo para la empresa, y con un $6 \%$ difunde la información relevante para las actividades de la organización y mantiene relaciones mutuamente con personas y organizaciones externas, y en menor proporción, el encargado de dar a conocer la opinión oficial frente a los temas que le competen y resuelve los disturbios personales que se producen entre los miembros.

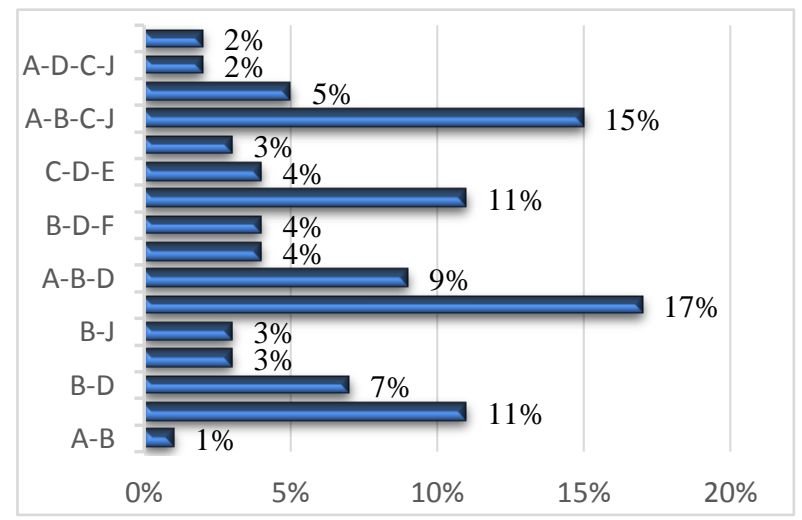

Figura 2. Habilidades Gerenciales

Fuente: Información suministrada por las empresas de la ciudad de Ocaña.
Las diferentes habilidades gerenciales que desarrollan las empresas, siendo el mayor. (Bojorquez, 2016)

$17 \%$ teniendo en cuenta que en este porcentaje hacen parte las tres habilidades más desarrolladas por los gerentes, siendo estas; la capacidad de gestionar los recursos, ya que permite tener un mayor control de estos y de igual forma tomar mejores decisiones, la capacidad de anticiparse a las necesidades del cliente, ya que el cliente cumple un papel fundamental dentro de la organización, teniendo en cuenta que este es la razón de ser de la empresa y por último la capacidad de desarrollar relaciones efectivas de intercambio con los demás, ayudando a que haya una buena comunicación y facilitando el intercambio de información. Otras de las habilidades que poseen los gerentes es la capacidad de identificar metas que permitan dirigir el rumbo de la empresa, siendo este es muy importante para el direccionamiento de la misma, permitiendo conocer hacia dónde ir, igualmente la capacidad de tomar decisiones sensatas, oportunas $\mathrm{y}$ efectivas, dado que de ellas dependerá en gran medida el desarrollo de la organización. (Heriquez, 2008)

Por otra parte, es necesario afirmar que la capacidad de obtener satisfacción personal del éxito del equipo y evaluar perfiles e identificar necesidades de desarrollo, fueron las de menor proporción, ya que son las menos desarrolladas por los diferentes gerentes. Por último, se puede decir que la capacidad para influir y cambiar la icta de otros, la capacidad de antıciparse a las tendencias económicas del futuro y la capacidad de proponer fórmulas de solución, son las habilidades 
que no tienen en cuenta los gerentes de las empresas, siendo estas de gran importancia para el direccionamiento de esta.

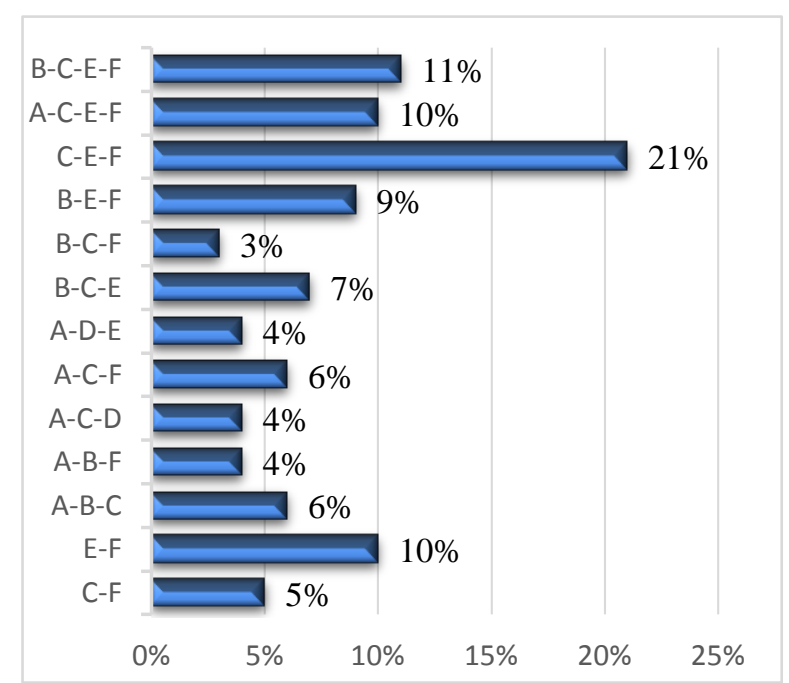

Figura 3.. Principales Actitud de los Gerentes de la Ciudad de Ocaña

Fuente: Información suministrada por las empresas de la ciudad de Ocaña.

En cuanto a las actitudes un $21 \%$, afirma que ejercen la autoconfianza, la cual les ayuda a desarrollar sus tareas con seguridad y afrontar circunstancias que se puedan presentar; de igual forma la motivación por el logro, el cual es importante para sobresalir en algo, superar los retos y alcanzar las metas, facilitando el crecimiento de la empresa, otras de las actitudes es el compromiso y sentido de identidad, siendo este fundamental para tomar consciencia de la importancia que tiene el cumplir con el desarrollo de su trabajo, asumiéndolo con responsabilidad y lealtad.

Por otra parte, cabe decir que las actitudes con menor proporción fueron la sensibilidad interpersonal y el autocontrol, siendo estos de gran importancia para el manejo de las emociones y comportamientos, no solo a nivel personal, sino también con los demás miembros y por último el trabajo bajo presión, la menos desarrollada por los gerentes.

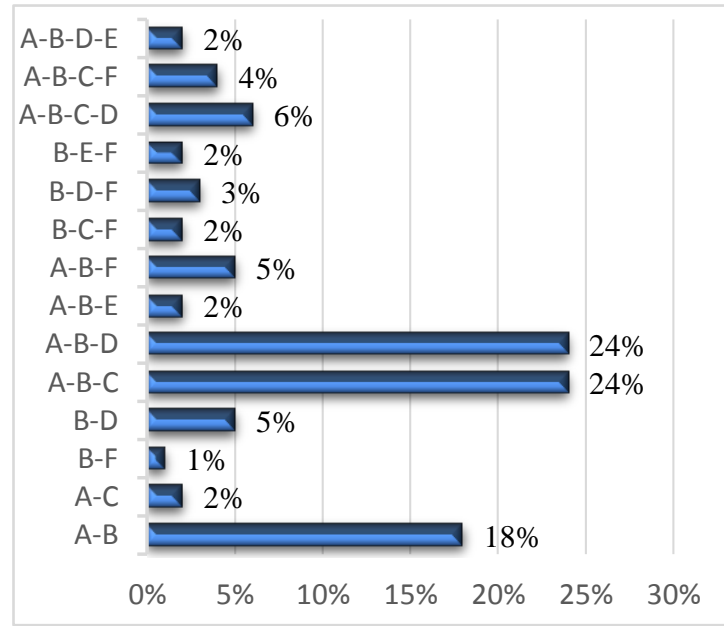

Figura 4. Destrezas de los Gerentes

- .ente: Información suministrada por las empresas de la ciudad de Ocaña.

En cuanto a las destrezas que desarrollan los encuestados se debe decir que el $24 \%$ eligieron tener la habilidad por el orden y la calidad, debido a que implica que las responsabilidades y funciones estén claramente asignadas en cada uno de los miembros del equipo; también como es la orientación al servicio al cliente, puesto que una adecuada orientación lleva a una mayor satisfacción para estos, con el compromiso de cumplir con sus pedidos, deseos y expectativas, implicando preocuparse por entender las necesidades de estos y dar solución a sus problemas, asimismo manifiestan tener el desarrollo de equipos, dirección de personas y liderazgo. Por otra parte, poseen la destreza de conocimientos, siendo estos adquiridos a través del ejercicio práctico o por la lectura permanente y finalmente la menos desarrolladas por los gerentes el ser analíticos y conceptuales. 


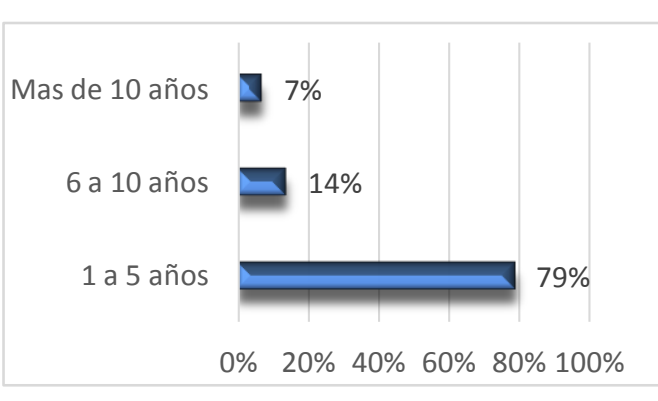

Figura 5. Años en el Cargo como Gerente

Fuente: Información suministrada por las empresas de la ciudad de Ocaña.

De los 254 encuestados, pertenecientes a las empresas de Ocaña el $79 \%$ tienen un

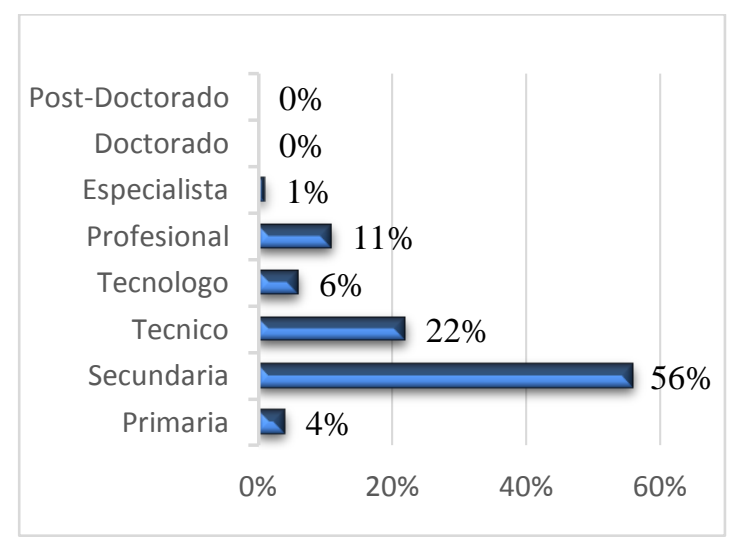

Figura 6. Formación que tiene en el desempeño de su cargo.

Fuente: Información suministrada por las empresas de la ciudad de Ocaña.

Siendo la formación educativa un fenómeno de gran relevancia, se pudo evidenciar que el $56 \%$ tiene una formación de secundaria, siendo este el mayor porcentaje, lo que quiere decir que un gran número de empresas son dirigidas por personas empíricas capaces de llevarlas al éxito y mantenerlas en crecimiento, tomando rango de tiempo entre 1 a 5 años en el cargo que desempeñan, cumpliendo con cada uno de los compromisos propuestos; El 14\% han estado en un periodo de 6 a 10 años en el cargo, es decir que han realizado un buen trabajo, lo que le ha facilitado seguir desarrollando su cargo.

Por otra parte, el $7 \%$ tienen más de 10 años, siendo estos propietarios o gerentes de las empresas, quienes son los encargados de dirigirlas y orientarlas al crecimiento; lo que significa que han tomado unas buenas decisiones, lo que les ha permitido permanecer dicho tiempo.

decisiones oportunas, ayudando a estas a permanecer en el mercado; como también el $22 \%$ siendo técnicos, permitiendo tener una base que facilite el desarrollo de las actividades; por otra parte un $11 \%$ son profesionales, lo que puede significar una ventaja importante, siendo esta una oportunidad para el desarrollo de los procedimientos y asimismo tomar mejores decisiones para orientar su empresa, el $6 \%$ tecnólogos, de igual forma un $4 \%$ de los encuestados tienen una formación de primaria, es decir no cuenta con una base de conocimientos, sin embargo han dirigido la empresa por un buen camino, lo que le has permitido permanecer en el mercado y el $1 \%$ especialista; sin embargo ninguno de los encuestados no cuentan con doctorado $\mathrm{y}$ post-doctorado. 


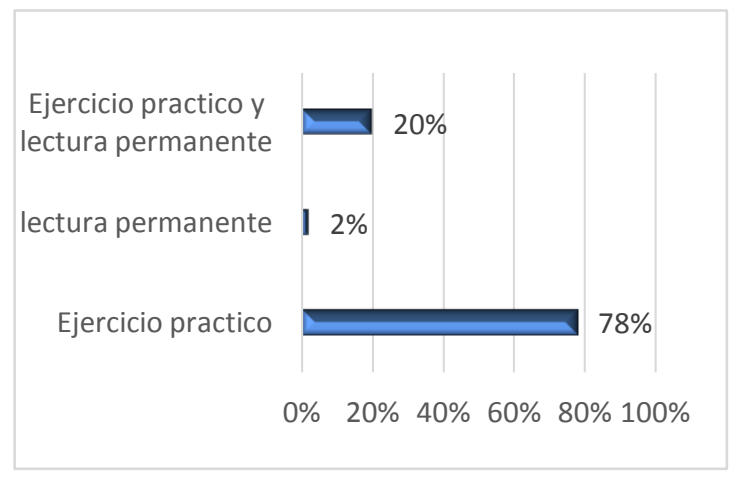

Figura 7. Conocimientos adquiridos.

Fuente: Información suministrada por las empresas de la ciudad de Ocaña.

De acuerdo a los resultados obtenidos, se observa que el mayor porcentaje es del $78 \%$, es decir, obtienen sus conocimientos a través del ejercicio práctico, como es la experiencia lograda en el transcurso del tiempo, siendo esta una base fundamental del conocimiento, por otra parte, un $20 \%$ manifestaron que tanto el ejercicio práctico como la lectura permanente; son partes importantes que ayudan adquirir una mayor preparación y conjuntamente ser un excelente líder, por ultimo con menor proporción del 2\% eligieron la lectura permanente.

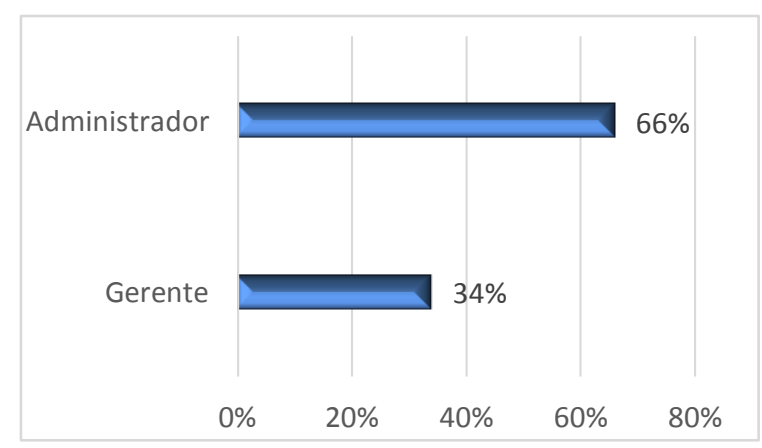

Figura 8. Cargo que Desempeña actualmente en la organización
Fuente: Información suministrada por las empresas de la ciudad de Ocaña.

Teniendo en cuenta la encuesta aplicada a 254 empresas legalmente constituidas de la ciudad de Ocaña, se debe decir que en cuanto al cargo que desempeñan, los encuestados respondieron que un $34 \%$ son gerentes, siendo la mayor parte de estos dueños y/o propietario de la empresa quienes son los que la dirigen, sin embargo un $66 \%$ son administradores, siendo estos los encargados del direccionamiento de las empresas, es decir los responsables de mantener y procesar toda la información necesaria para el crecimiento de esta.

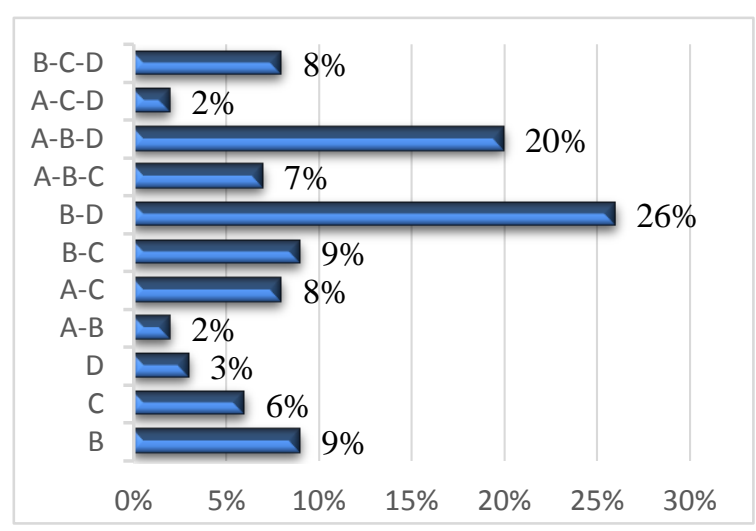

Figura 9. Estilo Gerencial

Fuente: Información suministrada por las empresas de la ciudad de Ocaña.

En cuanto a los estilos gerenciales, se puede observar que los gerentes manifestaron que un $26 \%$ poseen dos estilos, que son gerente como administrador, siendo este de gran importancia, ya que es el que se encarga de dirigir los miembros de la organización, el cual son parte fundamental e importante dentro de la misma, y asimismo toma decisiones de acuerdo a los objetivos organizacionales, buscado siempre los mejores resultados y el gerente orientado a las personas, es quien antepone a cada miembro las tareas, buscando siempre el bienestar de estos. Y los estilos menos 
desarrollados por los diferentes gerentes es el orientado a la tarea y como líder de equipo.

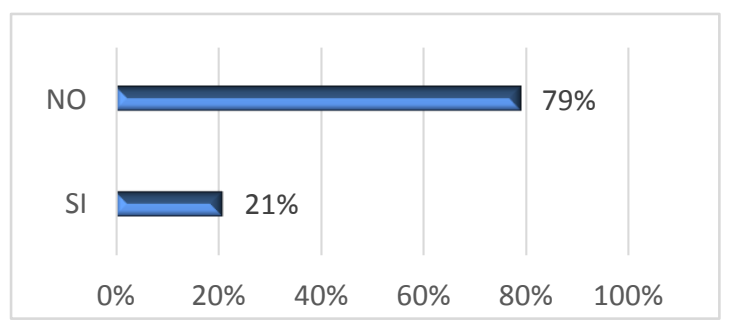

Figura 10. Estilo Gerencial en el Direccionamiento de los Recursos.

Fuente: Información suministrada por las empresas de la ciudad de Ocaña.

Considerando el desempeño en el estilo gerencial no influye en el direccionamiento de los recursos (Bracho, 2005), afirmando el $79 \%$, que es de gran importancia el manejo de este, ya que es la base fundamental de un buen gerente, por otra parte, un $21 \%$ eligieron que si influye el estilo gerencial en el manejo de los recursos, como es la eficiencia y la eficacia, puesto que su estilo es de orientación para el manejo de estos.

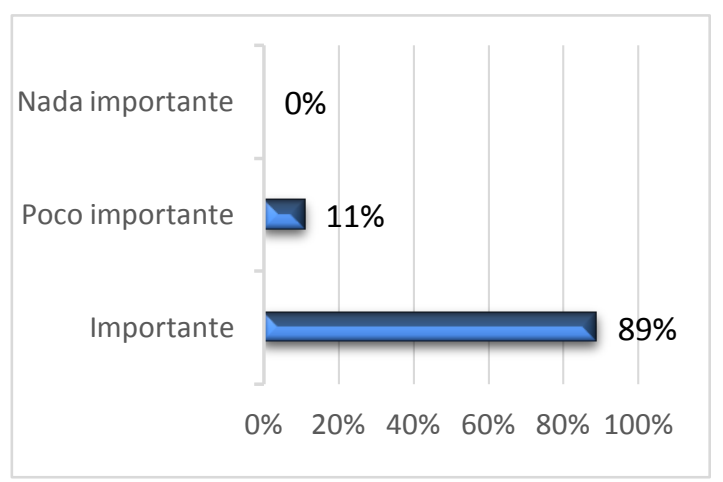

Figura 11. Importancia de la Toma de Decisiones

Fuente: Información suministrada por las empresas de la ciudad de Ocaña.

Los encuestados consideran que es de gran importancia tomar decisiones, esto lo afirma el $89 \%$, teniendo en cuenta que de acuerdo a las decisiones que se tomen dependerá en gran medida del direccionamiento y del éxito de la empresa, de igual forma, un $11 \%$ piensan que es poco importante tomar decisiones, lo que puede perjudicar los resultados que se quieren obtener, cabe decir que ninguno de los encuestados cree que al momento de tomar decisiones no se le da la importancia necesaria, es decir que esta cumple un papel significativo dentro de la organización.

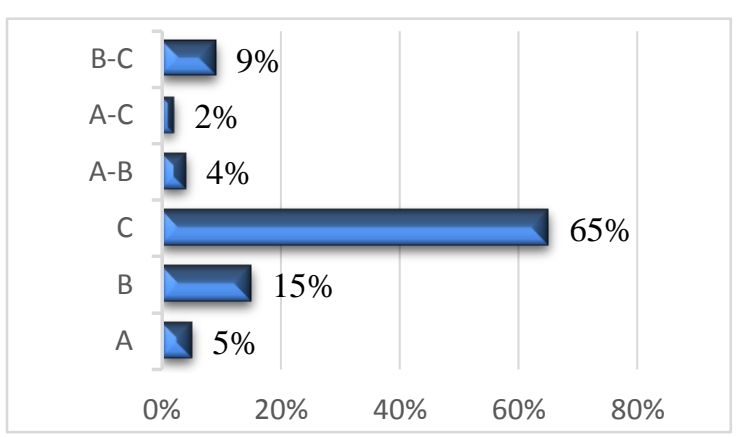

Figura 12. Tipos de decisiones

Fuente: Información suministrada por las empresas de la ciudad de Ocaña.

En las empresas de Ocaña los gerentes manifiestan que implementan las decisiones operativas o de regulación con un $65 \%$ es decir, gran número de los gerentes encuestados toman sus decisiones de modo habitual en la organización, siendo estas rutinarias, y con un $15 \%$ las decisiones tácticas o de pilotaje estas pueden ser repetitivos facilitando a los gerentes tomar decisiones.

Asimismo, un 5\% eligieron tomar decisiones estratégicas o de planificación sin embargo estas son de gran importancia; puesto que definen los objetivos de la organización las cuales son a largo plazo; ya que sus efectos son difícilmente reversibles teniendo en cuenta que algún error puede comprometer el desarrollo de la empresa. También cabe decir que el $9 \%$ implementan dos tipos de 
decisiones como son las tácticas y las operativas.

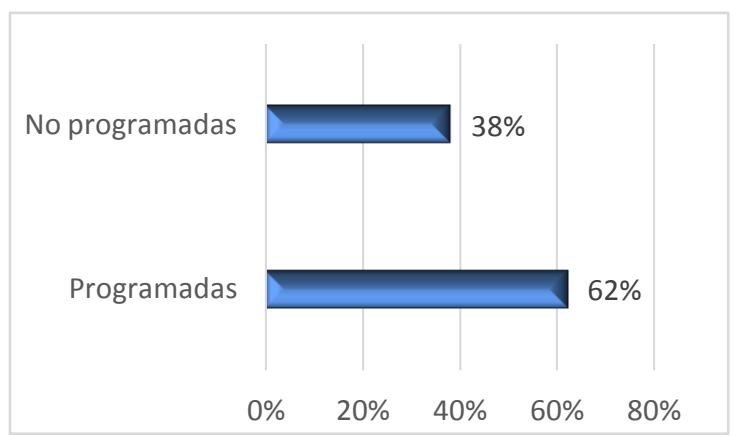

Figura 13. Características De las decisiones Decisiones Tomadas.

Fuente: Información suministrada por las empresas de la ciudad de Ocaña.

En cuanto a las decisiones tomadas en las diferentes empresas encuestadas, manifestaron que sus decisiones son programadas, es decir aquellas tomadas frecuentemente, repetitivas y se convierten en una rutina y no tienen la necesidad de diseñar ninguna decisión, simplemente se rige por la que ha seguido anteriormente, y un $38 \%$ afirmaron que sus decisiones son no programadas, ya que se toman en problemas o situaciones que se presentan con poca frecuencia.

\section{CONCLUSIONES.}

En cuanto a los roles, habilidades y destrezas de los gerentes de las empresas de la ciudad de Ocaña, se puede decir que estos desarrollan diferentes roles, que visualizan las oportunidades del entorno, habilidades como la capacidad de gestionar los recursos, de anticiparse a las necesidades del cliente y de desarrollar relaciones efectivas con los demás; además de adquirir destrezas como el orden y la calidad, orientación del servicio al cliente y desarrollo de equipo de trabajos orientándolos al direccionamiento de la organización; asimismo cabe resaltar que la formación, con la que cuentan los gerentes es limitada, es decir no tienen los estudios necesarios que requiere el cargo o las funciones, pero a pesar de ello han sabido dirigirla obteniendo conocimientos de manera práctica, sin tener claro su estilo gerencial.

El tipo de decisión tomada por los gerentes de las empresas de Ocaña son operativas, ya que sus decisiones son tomadas de acuerdo a lo que se presente en el día a día, es decir siendo estas rutinarias y en cuanto a sus estrategias, no se le da la importancia necesaria.

\section{BIBLIOGRAFÍA}

Bojorquez, F. (26 de Abril de 2016). LAS FUNCIONES GERENCIALES Y LOS ROLES GERENCIALES EN LA COMPETITIVIDAD DE LAS PYMES MANUFACTURERAS. CASO SINALOA. Red Internacional de Investigadores en Competitividad.

Bracho, A. (2005). Desempeño gerencial. COMPENDIUM , 5-18.

Duarte, 1. (2013). Habilidades Gerenciales y su importancia para el exito de una organización. Bogotá.

Ferrer, J., \& Clemenza, k. (2006). HABILIDADES GERENCIALES COMO FUNDAMENTO DE LA ESTRATEGIA COMPETITIVA EN LOS SECTORES DE ACTIVIDAD METALMECÁNICA VENEZOLANA. Obtenido de file:///F:/MATERIAL\%20DE\%20APO YO/JulianaFerrer.pdf

Guzman, M. (15 de Abril de 2014). Recuperado el 03 de Marzo de 2017, de 
http://www.eoi.es/blogs/madeon/2014/0

$4 /$

Heriquez, G. (2008). el rol del gerente de proyectos en el siglo XXI: actividades, habilidades, responsabilidades y criteris.

Herrera, M. (Enero de 2011). Formula Para Cálculo De La Muestra Poblaciones Finitas. Recuperado el 26 de Noviembre de 2016, de https://investigacionpediahr.files.wordpr ess.com/2011/01/formula-paracc3a1lculo-de-la-muestra-poblacionesfinitas-var-categorica.pdf

Larocca, H. (2010). Estilos gerenciales para el nuevo milenio. Barcelona, Buenos Aires. 